\title{
ESTUDO COMPARATIVO ENTRE OS DIAGNÓSTICOS MIELOGRÁFICO E ANÁTOMO-CIRÚRGICO NAS AFECÇõES RAQUEMEDULARES
}

\author{
Celso Pereira da Silva * \\ AfFonso SetTe JUNIOR **
}

O presente trabalho foi elaborado com a finalidade de apreciar a exatidão dos quadros mielográficos considerados típicos pela maioria dos autores. A idéia foi sugerida pelas freqüentes discordâncias verificadas entre aquêles quadros e os achados cirúrgicos.

\section{MATERIAL E METODOS}

Foram escolhidos sòmente casos em que os dados mielográficos pudessem ser comparados com os cirúrgicos; com êste critério foram reunidos 161 casos. O contraste usado em tôdas as mielografias foi o lipiodol, em quantidade máxima de $5 \mathrm{ml}$.

A fim de evitar idéias preconcebidas na interpretação dos mielogramas, o diagnóstico radiológico foi estabelecido independentemente dos resultados cirúrgicos; uma vez tabulados, os diagnósticos mielográficos foram relacionados com os achados operatórios. A seguir os casos foram agrupados de acôrdo com os diagnósticos mielográficos, a saber: hérnia de núcleo pulposo, hipertrofia do ligamento amarelo, aracnoidite, angioma, bloqueio por tumor intramedular, bloqueio por tumor extradural, mielogramas atípicos e mielogramas não conclusivos.

Hérnia do núcleo pulposo - Imagem típica mostrando defeito unilateral da coluna lipiodolada ao nivel do disco intervertebral, observado nas incidências ânteroposterior e de perfil, com o paciente em decúbito ventral (fig. 1).

Hipertrofia do ligamento amarelo - Imagem típica mostrando estrangulamento da coluna de lipiodol ao nivel do disco intervertebral (imagem em ampulheta), verificada na incidencia ântero-posterior e com o paciente em decúbito dorsal (fig. 2).

Aracnoidite - Imagens variadas, como sejam, parada permanente do lipiodol em gotículas disseminadas por uma extensão variável do canal raqueano, ou em cordões também dispostos por uma extensão variável (fig. 3).

Angioma - Imagem típica sob a forma de cordões dispostos em geral longitudinalmente, apresentando dilatações saculares, o conjunto lembrando o esofagograma em caso de varizes do esôfago (fig. 4).

Bloqueio por tumor intramedular - Imagem típica sob a forma de cordões situados de um lado e outro do canal raqueano apresentando, cada um, uma série de expansões laterais ou linguetas em correspondencia com os espacos intervertebrais (fig. 5).

Trabalho do Serviço de Neurologia da Escola Paulista de Medicina (Prof. Paulino W. Longo), apresentado às Jornadas Radiológicas (Curitiba, 1954). * Neuroradiologista; ** Neurocirurgião. 


\begin{tabular}{|c|c|c|c|c|c|c|c|c|c|c|}
\hline $\begin{array}{r}\text { Diagnóstico } \\
\text { mielográfico }\end{array}$ & 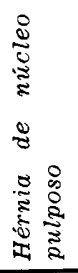 & 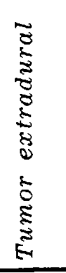 & 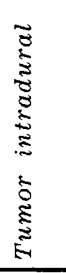 & 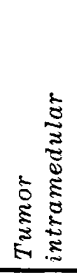 & 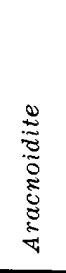 & 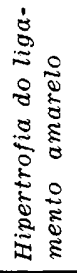 & 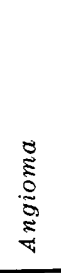 & 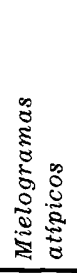 & 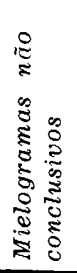 & $\begin{array}{l}\sim \\
0 \\
+ \\
0 \\
i\end{array}$ \\
\hline $\begin{array}{c}\text { Hérnia de núcleo } \\
\text { pulposo } \ldots . . .\end{array}$ & $29^{*}$ & 3 & 1 & 1 & - & 3 & - & 4 & 2 & 43 \\
\hline $\begin{array}{c}\text { Tumor extradu- } \\
\text { ral } \ldots \ldots \ldots \ldots\end{array}$ & 2 & $16^{*}$ & 2 & 3 & 2 & - & - & 1 & 1 & 27 \\
\hline $\begin{array}{c}\text { Tumor intradu- } \\
\text { ral } \ldots \ldots \ldots \ldots\end{array}$ & - & 7 & $10^{*}$ & 3 & -- & -- & - & 1 & 1 & 22 \\
\hline $\begin{array}{c}\text { Tumor intrame- } \\
\text { dular } \quad \ldots \ldots \ldots\end{array}$ & $-\cdots$ & 3 & 2 & $4^{*}$ & - & - & - & 3 & - & 12 \\
\hline Aracnoidite. & 2 & 3 & 3 & 3 & $10^{*}$ & - & - & 7 & 3 & 31 \\
\hline $\begin{array}{l}\text { Hipertrofia do li. } \\
\text { gamento ama- } \\
\text { relo } \ldots \ldots \ldots \ldots\end{array}$ & 6 & $-\cdots$ & - & - & - & 2 & - & 1 & - & 9 \\
\hline $\begin{array}{l}\text { Protrusão de nú- } \\
\text { cleo pulposo }+ \\
\text { hipertrofia lig. } \\
\text { amarelo } \ldots . . .\end{array}$ & 3 & - & - & 一 & -- & 2 & - & - & - & 5 \\
\hline $\begin{array}{l}\text { Protrusão de nú- } \\
\text { cleo pulposo }\end{array}$ & 2 & - & -- & $\cdots$ & 1 & - & -- & -- & - & 3 \\
\hline Angioma $\ldots \ldots$ & - & - & - & - & - & 1 & $3^{*}$ & - & - & 4 \\
\hline $\begin{array}{c}\text { Tumor intra } e \\
\text { extradural } \ldots\end{array}$ & - & 1 & 1 & - & - & - & -- & - & - & 2 \\
\hline $\begin{array}{l}\text { Canal raqueano } \\
\text { malformado }\end{array}$ & -- & 1 & - & $\cdots$ & - & - & - & - & - & 1 \\
\hline $\begin{array}{l}\text { Negativo cirùr- } \\
\text { gicamente } \ldots\end{array}$ & 1 & - & - & 1 & - & - & -- & - & - & 2 \\
\hline Total ..... & 45 & 34 & 19 & 15 & 13 & 8 & 3 & 17 & 7 & 161 \\
\hline
\end{tabular}

Quadro 1 - Comparação entre os diagnósticos radiológico e cirúrgico. * Número de casos com diagnóstico radiológico e cirúrgico concordantes. 

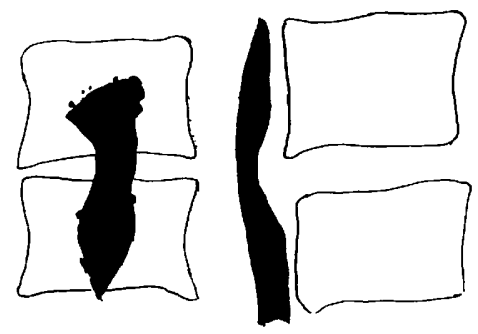

Fig. 1 - Imagem típica de hérnia do núcleo pulposo: à esquerda incidência ântero-posterior; $\grave{a}$ direita incidência de perfil.

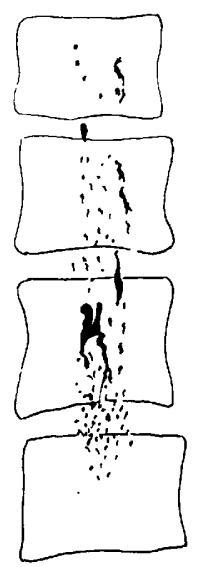

Fig. 3 - Imagem típica de aracnoidite.

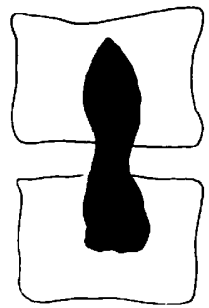

Fig. 2 - Imagem tipica de hipertrofia do ligamento amarelo.

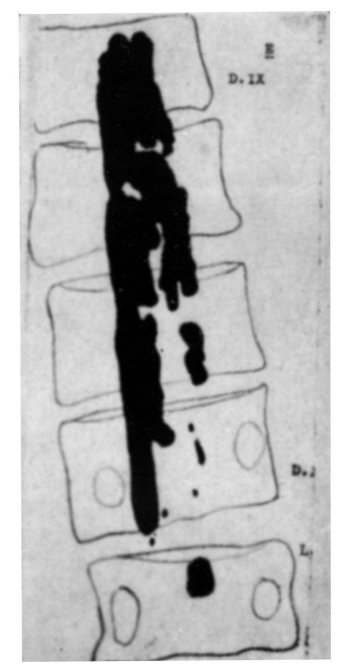

Fig. 4 - Inagem tipica

Fig. 5 - Imagem típica de tumor intramedular. 
Bloqueio por tumor intradural - Imagem típica sob a forma de uma coifa ou domo ou casquete, que desenha o pólo correspondente do tumor (fig. 6).

Bloqueio por tumor extradural - Imagem típica de parada do lipiodol com limite inferior grosseiramente linear ou festonado; em alguns casos os bordos laterais apresentam defeitos em estrias (fig. 7).

Mielogramas atípicos - São os que mostram imagem nitidamente patológìca, mas não classificável sob qualquer dos quadros acima descritos (fig. 8).

Mielogramas não conclusivos - Imagem mielográfica não permitindo conclusão sôbre seu caráter normal ou patológico.
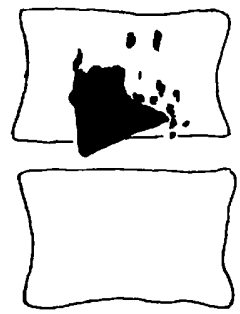

Fig. 6 - Imagem típica de tumor intradural.

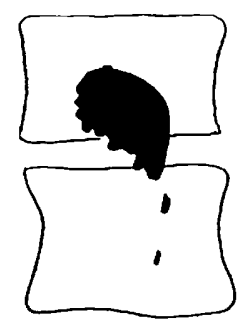

Fig. 7 - Imagem tipica de tumor extradural.

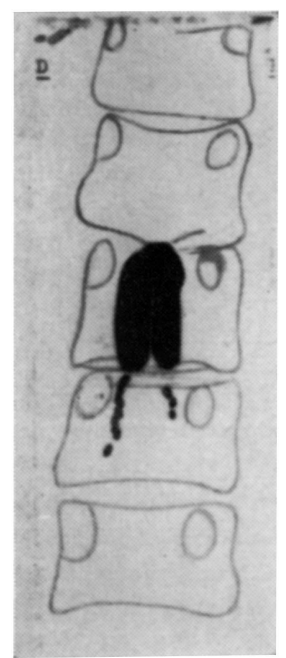

Fig. 8 - Mielograma atipico.

\section{RESULTADOS}

Os resultados obtidos vão expostos no quadro 1, no qual estão consignados, nas colunas verticais, os achados anátomo-cirúrgicos correspondentes a cada grupo de casos diagnosticados pela mielografia, figurando, nos pés das colunas, o total de casos de cada um dêstes grupos; as colunas horizontais mostram, para cada grupo de casos com o mesmo diagnóstico anátomo-cirúrgico, os diagnósticos mielográficos prèviamente feitos. Na última casa de cada coluna horizontal está o número total de casos de cada grupo com o mesmo diagnóstico anátomo-cirúrgico.

Hérnia do núcleo pulposo - Foram diagnosticados radiologicamente 45 casos, dos quais 29, ou seja $64,4 \%$, foram confirmados pela intervenção cirúrgica. Nos 16 casos restantes a imagem mielográfica considerada típica de hérnia do núcleo pulposo foi determinada por entidades anátomo-cirúrgicas várias: hipertrofia do ligamento amarelo (6 casos), aracnoidite (2), tumor extradural (2), protrusão discal associada à hjpertrofia do ligamento amarelo (3), protrusão simples do disco (2); houve um caso com achado operatório negativo. 
Sob o aspecto anátomo-cirúrgico, a série abrange 43 casos de hérnia de disco intervertebral, dos quais 29 com diagnóstico mielográfico concordante $(29: 43$, ou seja $67,4 \%$ ). Os 14 casos anátomo-cirúrgicos mostraram imagem mielográfica de hipertrofia do ligamento amarelo (3), tumor intramedular (1), tumor intradural (1), tumor extradural (3), atipicas (4); imagens não conclusivas (2).

Hipertrofia do ligamento amarelo - Foram diagnosticados radiologicamente 8 casos, dos quais em 2 houve concordância anátomo-cirúrgica. Nos restantes, os diagnósticos anátomo-cirúrgicos foram de hipertrofia do ligamento amarelo associada a protrusão discal (2), de hérnia do núcleo pulposo (3) e de angioma (1).

O diagnóstico anátomo-cirúrgico de hipertrofia do ligamento amarelo foi feito em 9 casos, dos quais em 2 houve concordância com o diagnóstico mielográfico. Nos 7 casos discordantes, 6 tiveram o diagnóstico mielográfico de hérnia do núcleo pulposo, havendo um mielograma atípico.

Tumor extradural - Foram diagnosticados radiologicamente 34 casos, dos quais 16 confirmados pelo ato cirúrgico $(16: 34$, ou seja $47,6 \%)$. Nos 18 casos restantes os diagnósticos anátomo-cirúrgicos foram de tumor intradural (7), tumor intramedular (3), tumor intra e extradural (1), aracnoidite (3), hérnia do núcleo pulposo (3) e malformação do canal raqueano (1).

O diagnóstico anátomo-cirúrgico de tumor extradural foi feito em 27 casos, 16 dos quais com diagnóstico mielográfico concordante. Os outros 11 casos mostraram imagem mielográfica de tumor intramedular (3), de hérnia do núcleo pulposo (2), de aracnoidite (2), de tumor intradural (2), atipica (1), imagem não conclusiva (1).

Tumor intradural - Foram diagnosticados radiologicamente 19 casos, dos quais 10 concordantes com o diagnóstico anátomo-cirúrgico $(10: 19$, ou seja $52,6 \%)$. Nos 9 casos restantes os diagnósticos anátomo-cirúrgicos foram de tumor extradural (2), tumor intramedular (2), tumor intra e extradural (1), aracnoidite (3) e hérnia do núcleo pulposo (1).

O diagnóstico anátomo-cirúrgico de tumor intradural foi feito em 22 casos, dos quais 10 com diagnóstico mielográfico concordante. Os outros 12 casos apresentaram imagem mielográfica de tumor extradural (7), tumor intramedular (3), atipica (1); mielograma não conclusivo (1).

Tumor intramedular - Foram diagnosticados radiológicamente 15 casos, dos quais 4 foram confirmados pelo ato cirúrgico $(4: 15$, ou seja $26,6 \%)$. Nos 11 casos restantes os diagnósticos anátomo-cirúrgicos foram de tumor intradural (3), tumor extradural (3), aracnoidite (3), hérnia do núcleo pulposo (1). havendo um caso com achado cirúrgico negativo.

o diagnóstico anátomo-cirúrgico de tumor intramedular foi feito em 12 casos, dos quais 4 concordantes com o diagnóstico mielográfico. Os outros 8 casos apresentaram imagem mielográfica de tumor extradural (3), tumor intradural (2); atipica (3).

Aracnoidite - Foram diagnosticados radiologicamente 13 casos, dos quais 10 concordantes com os achados anátomo-cirúrgicos $(10: 13$, ou seja $76,9 \%)$. Nos 3 casos discordantes os diagnósticos anátomo-cirúrgicos foram de protrusão discal (1) e tumor extradural (2).

O diagnóstico anátomo-cirúrgico de aracnoidite foi feito em 31 casos, dos quais 10 concordantes com o mielograma. Os 21 casos restantes apresentavam imagem mielográfica de tumor intramedular (3), tumor intradural (3), tumor extradural (3), hérnia do núcleo pulposo (2), atípica (7); imagem não conclusiva (7).

Angiomas - Foram diagnosticados radiologicamente 3 casos, todos concordantes com os achados anátomo-cirúrgicos. 
O diagnóstico neurocirúrgico de angioma foi feito em 4 casos, sendo 3 concordantes com o mielograma; em um fôra feito diagnóstico mielográfico de hipertrofia do ligamento amarelo.

Mielogramas atipicos - Foram registrados em 17 casos (17:161, ou seja 10,6 \% $\%^{\circ}$, nos quais o exame anátomo-cirúrgico mostrou tratar-se de aracnoidite (7), hérnia do núcleo pulposo (4), hipertrofia do ligamento amarelo (1), tumor extradural (1), tumor intradural (1) e tumor intramedular (3).

Mielogramas nüo conclusivos - Foram obtidos em 7 casos $(7: 161$, ou seja $4,3 \%)$, nos quais o diagnóstico anátomo-cirúrgico foi de aracnoidite (3), hérnia do núcleo pulposo (2), tumor extradural (1) e tumor intradural (1).

Análise estatistica dos resultados:- Procuramos verificar, por um teste de diferença de proporçōes, a possibilidade de ser explicada pela flutuação causal a diferença encontrada entre o número de casos com determinado diagnóstico mielográfico e o número de casos com o mesmo diagnóstico feito anátomo-cirùrgicamente.

Nos casos de hérnia do núcleo pulposo, tumor extradural, tumor intradural e aracnoidite, essas diferenças não foram significativas. Entretanto, nos casos de tumor intramedular e de hipertrofia do ligamento amarelo, o nivel de significância de $5 \%$ foi ultrapassado. Conseqüentemente, o material estudado permite a afirmacão de que o diagnóstico mielográfico de tumor intramedular e de hipertrofia do ligamento amarelo é pouco seguro, podendo as imagens radiológicas consideradas tipicas ser determinadas por outras lesões.

\section{COMENTARIOS}

Para melhor interpretação dêstes resultados reunimos, no quadro 2, os dados referentes à concordância entre os diagnósticos anátomo-cirúrgico e mielográfico, bem como, para cada grupo, o acêrto e êrro mielográfico: a concordância é dada pela relação entre o número de casos com diagnóstico mielográfico comprovado e o número de casos diagnosticados anátomocirùrgicamente; o acêrto mielográfico, pela relação entre o número de casos com diagnóstico mielográfico comprovado e o número total de casos com o mesmo diagnóstico mielográfico; o êrro mielográfico, pela relação entre o número de casos com diagnóstico mielográfico não comprovado e o número total de casos com o mesmo diagnóstico mielográfico.

A importância de levarmos em consideração o acêrto mielográfico e a concordância pode ser bem exemplificada no grupo das aracnoidites, onde um acêrto mielográfico de $77 \%$ sugere uma precisão diagnóstica elevada; entretanto, a concordância mostra que, no total de 31 casos, apenas 13 foram diagnosticados pela mielografia, ou seja $32 \%$.

Não estão incluídos no quadro 2 alguns diagnósticos anátomo-cirúrgicos contidos no quadro 1 e que não apresentam concordância com qualquer das imagens ou diagnósticos mielográficos existentes, mas cuja análise oferece algum interêsse. Assim temos:

* Agradecemos ao Dr. Isnard Reis Filho a colaboração que nos prestou, fazendo a análise estatísticá dos resultados. 
Cinco casos de protrusão de núcleo pulposo associada à hipertrofia do ligamento amarelo, dos quais 3 com diagnóstico mielográfico de hérnia do núcleo pulposo e 2 de hipertrofia do ligamento amarelo; três casos de protrusão de núcleo pulposo, dos quais dois com diagnóstico mielográfico de hérnia do núcleo pulposo e um de aracnoidite.

Os dois grupos acima referidos compreendem 8 casos com o diagnóstico anátomo-cirúrgico de protrusão discal, simples ou associada à hipertrofia do ligamento amarelo; em 5 dêles o diagnóstico mielográfico foi o de hérnia do núcleo pulposo; em 2 o exame mielográfico levou ao diagnóstico de hipertrofia do ligamento amarelo, tendo a intervenção cirúrgica, aliás, confirmado a existência de hipertrofia do ligamento amarelo; em um o exame mielográfico sugeriu o diagnóstico de aracnoidite. Assim, em nossos casos, a mielografia não permitiu fazer a diferenciação que existe, sob o ponto de vista anátomo-cirúrgico, entre protrusão e hérnia de núcleo pulposo; do mesmo modo não permitiu fazer a diferenciação entre a protrusão simples do disco e a protrusão associada à hipertrofia do ligamento amarelo.

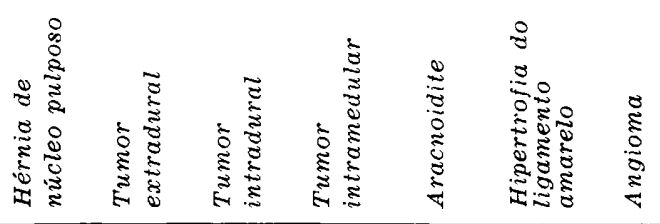

Número de casos comprovados anátomo-cirùrgicamente $\ldots .$.

Número de casos com diagnóstico mielográfico ............

Número de casos com diagnóstico mielográfico comprovado ..

Número de casos com diagnóstico mielográfico não comprovado

Concordância entre os diagnóstico mielográfico e anátomo-cirúrgico $\ldots \ldots \ldots \ldots \ldots \ldots$.
43

27

45

34

29

16

10

4

10

2

3

16

16

\begin{abstract}
18
\end{abstract}

9

11

3

6

0

\begin{tabular}{|c|c|c|c|c|c|c|c|}
\hline $\begin{array}{l}\text { Concordância entre os diagnósti- } \\
\text { co mielográfico e anátomo-ci- } \\
\text { rúrgico } \ldots \ldots \ldots \ldots \ldots \ldots \ldots \ldots\end{array}$ & $67 \%$ & $59 \%$ & $45 \%$ & $33 \%$ & $32 \%$ & $22 \%$ & $75 \%$ \\
\hline Acêrto radiológico $\ldots$. & $64 \%$ & $47 \%$ & $53 \%$ & $27 \%$ & $77 \%$ & $25 \%$ & $100 \%$ \\
\hline Erro radiológico .. & $36 \%$ & $53 \%$ & $47 \%$ & $73 \%$ & $23 \%$ & $75 \%$ & $0 \%$ \\
\hline
\end{tabular}

Quadro 2 - Concordância entre os diagnósticos anátomo-cirúrgicos e mielográficos. Dados para o estudo do acêrto e êrro radiológico. 
Reunindo os 5 casos de diagnóstico anátomo-cirúrgico de hipertrofia do ligamento amarelo associada à protrusão discal, com os 9 casos de hipertrofia simples do ligamento amarelo, teremos 14 casos de hipertrofia do ligamento amarelo; em 4 dêstes casos houve concordância com o mielograma quanto à hipertrofia ligamentar; em 10 houve discordância, com 9 diagnósticos mielográficos de hérnia do núcleo pulposo e uma mielografia atípica. Portanto, em nossos casos, o diagnóstico mielográfico de hipertrofia do ligamento amarelo correspondeu, na maioria das vêzes, ao achado anátomo-cirúrgico de protrusão ou de hérnia discal.

No quadro 2 também não foram incluídos: dois casos de tumor intra e extradural diagnosticados pelos achados anátomo-cirúrgicos e nos quais o mielograma foi o de uma ou da outra entidade; um caso de malformação do canal raqueano, diagnosticado anátomo-cirùrgicamente e no qual o exame mielográfico sugerira a existência de tumor extradural. Também não estão incluídos nesse quadro dois casos cirùrgicamente negativos, nos quais o exame mielográfico sugerira, respectivamente, a existência de hérnia de núcleo pulposo e de tumor intramedular.

A título de referência reunimos as percentagens de concordância entre o diagnóstico mielográfico e o anátomo-cirúrgico, obtidas por vários autores, no que diz respeito à hérnia do núcleo pulposo:

\begin{tabular}{|c|c|c|}
\hline A ut ores & $\begin{array}{c}\text { Concordância em } \\
\text { percentagem }\end{array}$ & $\begin{array}{l}\text { Número de } \\
\text { casos }\end{array}$ \\
\hline Leader e Marvin $^{9}$ & 86 & 414 \\
\hline$\ldots \ldots \ldots \ldots \ldots \ldots \ldots$ & 88,1 & 300 \\
\hline Scoville, Moretz e Hankins ${ }^{15} \ldots \ldots \ldots \ldots \ldots \ldots \ldots$ & 88 & 200 \\
\hline 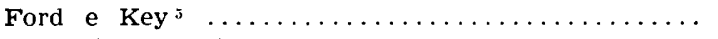 & 83,5 & 164 \\
\hline Borrelli e Maglione ${ }^{1} \ldots \ldots \ldots \ldots \ldots \ldots \ldots \ldots \ldots$ & 85 & 132 \\
\hline Ford, Ransey, Holt e Key $^{6} \ldots \ldots \ldots \ldots \ldots \ldots \ldots$ & 60 & 100 \\
\hline$\ldots \ldots \ldots \ldots \ldots \ldots \ldots \ldots$ & 79,6 & 64 \\
\hline Lange $e$ Odegaard $^{s} \ldots \ldots \ldots \ldots \ldots \ldots \ldots \ldots \ldots$ & 88,8 & 63 \\
\hline Echlin, Silverstone e Schribner ${ }^{3} \ldots \ldots \ldots \ldots \ldots \ldots$ & 91,6 & 60 \\
\hline Pereira da Silva e Sette Junior $\ldots \ldots \ldots \ldots \ldots \ldots$ & 67,4 & 43 \\
\hline Peyton e Simmons ${ }^{13} \ldots \ldots \ldots \ldots \ldots \ldots \ldots \ldots \ldots$ & 97,6 & 42 \\
\hline Stinchfield e Sinton $^{17} \ldots \ldots \ldots \ldots \ldots \ldots \ldots \ldots \ldots$ & 80 & 36 \\
\hline Schnitker e Bruce ${ }^{14} \ldots \ldots \ldots \ldots \ldots \ldots \ldots \ldots \ldots \ldots$ & 85,7 & 35 \\
\hline Craig, Svien, Dodge e Camp ${ }^{2} \ldots \ldots \ldots \ldots \ldots \ldots \ldots$ & 85,1 & 27 \\
\hline Wilson e Ilfeld ${ }^{19} \ldots \ldots \ldots \ldots \ldots \ldots \ldots \ldots \ldots \ldots$ & 91,6 & 12 \\
\hline
\end{tabular}

Analisando as causas de êrro na interpretação dos mielogramas, Scoville, Moretz e Hankins ${ }^{1 i}$ e Orley ${ }^{12}$ assinalam hérnias múltiplas, discos ocultos, fundo de saco dural muito alto, canal raqueano anormalmente amplo, saco dural congênitamente estreitado, defeitos pós-operatórios, aderências, saliência normal dos discos intervertebrais tomada como hérnia discal (principalmente na região lombar), defeito de enchimento produzido por hérnia discal muito volumosa interpretado como imagem de tumor (principalmente na 
incidência ântero-posterior e no caso de bloqueio completo), hipertrofia unilateral do ligamento amarelo dando defeito unilateral como na hérnia discal, hiperplasia da gordura extradural ou veias epidurais anômalas, produzindo defeito de enchimento tipo hérnia do núcleo pulposo. $\mathrm{O}$ mesmo defeito pode ser ainda causado por lesōes vertebrais, como esporão espondilítico posterior.

Com relação aos tumores muito poucas referências conseguimos encontrar. Craig, Svien, Dodge e Camp 2, em 24 casos com diagnóstico mielográfico de tumor, sem precisar a natureza, obtiveram concordância de 100 ; com o achado operatório. Tucker ${ }^{18}$, em 196 mielografias com pantopaque, diferencia tumores (intradural, extradural, intramedular) entre si e da hérnia de núcleo pulposo, descreve as imagens mielográficas e relata os achados anátomo-patológicos, sem referir as percentagens de concordância.

Em sintese, as percentagens de concordância entre o mielograma e o diagnóstico anátomo-cirúrgico encontradas na literatura, referentes quase que exclusivamente à hérnia de núcleo pulposo, são em geral superiores às que obtivemos. Acôrdo existe, porém, no que se refere ao fato de que uma dada imagem mielográfica considerada típica pode corresponder a vários quadros anátomo-cirúrgicos.

Finalizando, julgamos oportuno salientar o trabalho pioneiro de Sicard e Forestier ${ }^{16}$, que demonstraram a inocuidade e a possibilidade do uso subaracnóideo do lipiodol, criando, assim, o método ao qual denominaram "prova do lipiodol subaracnóideo"; êstes autores já tinham assinalado as dificuldades que podem surgir na interpretação das imagens mielográficas e a necessidade dos resultados serem confrontados com dados clínicos, com os elementos fornecidos pelo exame do liqüido cefalorraqueano, pela radiografia simples e pela mieloscopia. A confiança de Sicard e Forestier em seu método os levou a afirmar que nos casos em que a mielografia localizar um processo em nível diferente indicado pela clínica, o neurologista deve orientar-se de preferência pela indicação mielográfica; entretanto, a experiência mostrou que a mielografia não pode ser considerada de modo tão absoluto.

A conclusão final é a de que a mielografia, além dos dados que fornece sôbre a localização e extensão dos bloqueios dos espaços subaracnóideos, pode contribuir para o diagnóstico da natureza das lesões. Entretanto, como cada uma das imagens mielográficas consideradas típicas pode corresponder a diagnósticos anátomo-cirúrgicos diferentes, assim como determinado diagnóstico anátomo-cirúrgico pode produzir quadros mielográficos diversos, o valor da contribuição da imagem mielográfica para o diagnóstico da natureza das lesões não é absoluto, tendo apenas valor relativo, variável para cada grupo de casos de um determinado diagnóstico.

Assim, julgamos ser mais acertado substituir a designação de "imagem mielográfica típica" pela de "imagem mielográfica sugestiva" de determinado processo. Tal é a conduta que adotamos em nossos relatórios neuro-radiológicos. 


\section{RESUMO}

O presente trabalho visa apreciar a exatidão das imagens mielográficas consideradas tipicas pela maioria dos autores.

Para êste fim os mielogramas de 161 casos operados foram divididos em 9 grupos, dos quais 7 (no total de 137 casos) com imagens consideradas tipicas e 2 (contendo 24 casos) com imagens atípicas ou não conclusivas. Os diagnósticos mielográficos foram, a seguir, confrontados com os achados anátomo-cirúrgicos, obtendo-se uma concordância de $75 \%$ para o grupo dos angiomas, de $67,4 \%$ para o de hérnia de núcleo pulposo, de $59,2 \%$ para o de tumor extradural, de $45,4 \%$ para o de tumor intradural, de $33,3 \%$ para o de tumor intramedular, de $32,2 \%$ para o de aracnoidite e de $22,2 \%$ para o de hipertrofia do ligamento amarelo. A parte discordante em cada grupo abrangeu diagnósticos anátomo-cirúrgicos os mais variados. $\mathrm{A}$ análise estatística mostrou significância apenas para os grupos de tumor intramedular e hipertrofia do ligamento amarelo.

A conclusão é a de que a imagem mielográfica pode contribuir para o diagnóstico da natureza das lesões obstrutivas dos espaços subaracnóideos; a sua exatidão porém não é absoluta, mas apenas relativa, sendo variável para cada entidade. A denominação habitualmente usada de "imagem típica" deve, por isso, ser substituida pela de "imagem sugestiva" de um determinado processo lesional.

\section{SUMMARY}

Myelographic and anatomo-surgical diagnosis in spinal lesions. Comparative study.

This paper is based on a review of 161 cases, in which lipiodol myelography had been done and which were operated upon, thereby so affording an accurate anatomic diagnosis for comparison with the myelographic findings. The latter were divided into nine groups: seven including cases with myelographic aspects considered typical (137 cases) and two with atypical or inconclusive myelograms (24 cases). For each one of these myelographic group, it was verified agreement with the surgical findings in percentages such as follows: angioma, 75\%; ruptured intervertebral disc, 67.4\%; extradural tumor, 59.2\%; intradural tumor, $45.4 \%$; intramedullar tumor, $33.3 \%$; arachnoiditis, $32.2 \%$; hypertrophy of ligamentum flavum, $22.2 \%$.

Discordant cases showed that the same myelographic aspect may be related to various anatomosurgical diagnosis. Statistical approaches have shown significance only for intramedullar tumor and hypertrophy of ligamentum flavum groups. Some surgical findings could not be related to any myelographic aspect.

Conclusion is that myelographic aspects have some value to predict the nature of the lesions, but not in a definite way, due to the possibility of 
discordances as shown above. So, it seems more adequate to speak of "suggestive myelographic aspects" instead of "typical aspects".

\section{REFERENCIAS}

1. BORRELLI, F. J.; MAGLIONE, A. A. - The importance of myelography in spinal pathology: analytical study of 150 cases. Am. J. Roentgenol., 76:273, 1956. 2. CRAIG, W. M.; SVIEN, H. J.; DODGE, H. W.; CAMP, J. R. - Intraspinal lesions masquerading protruded lumbar intervertebral discs. J.A.M.A., 149:250, 1952.3. ECHLIN, F. A.; SILVERSTONE, B.; SCHRIBNER, W. E. - Bilateral and multiple ruptured discs as one cause of persistent symptoms following operation for herniated disc. Surg., Gynecol. \& Obst., 83:485, 1946. 4. EPSTEIN, B. S. - Low back pain associated with varices of the epidural veins simulating herniation of the nucleus pulposus. Am. J. Roentgenol., 57:736, 1947. 5. FORD, L. T.; KEY, J. A. - An evaluation of myelography in diagnosis of intervertebral disc lesions in the low back pain. J. Bone a. Joint Surg., 32:257, 1950. 6. FORD, L. T.; RANSEY, R. H.; HOLT, E. P.; KEY, J. A. - An analysis of one hundred consecutive lumbar myelograms followed by disc operation for relief of low back pain and sciatica. Surgery, 32:961, 1952. 7. HEISER, S.; SWYER, A. J. - Myelography in spinal metastases. Radiology, 62:695, 1954. 8. LANGE, J.; ODEGAARD, H. - Abrodil myelography in herniated disk in the lumbar region. Radiology, 57:186, 1951. 9. LEADER, S. A.; MARVIN, J. R. - The value of pantopaque myelography in the diagnosis of herniation of the nucleus pulposus in the lumbo-sacral spine: a report of 500 cases. Am. J. Roentgenol., 69:231, 1953. 10. MAC CARTY, W. C.; LANE, F. W. - Pitfalls of myelography. Radiology, 65:663, 1955. 11. MALTBY, G. L.; PENDERGRASS, R. C. - Pantopaque myelography: diagnostic errors and review of cases. Radiology, 47: 35, 1946. 12. ORLEY, A. - Neuroradiology. Charles C. Thomas, Springfield, 1949. 13. PEYTON, W. T.; SIMMONS, D. R. - Herniation of the intervertebral disc: analysis of ninety cases. Arch. Surg., 55:271, 1947. 14. SCHNITKER, M. T.; BOOTH, G. T. - Pantopaque myelography for protruded disks of the lumbar spine. Radiology, 45:370, 1945. 15. SCOVILlE, W. B.; MORETZ, W. H.; HANKINS, W. D. Discrepancies in myelography: estatistical survey of 200 operative cases undergoing Pantopaque myelography. Surg., Gynecol. \& Obst., 86:559, 1948. 16. SICARD, J. A.; FORESTIER, J. - Diagnostique et Thérapeutique par le Lipiodol. Masson et Cie., Paris, 1928, págs. 125-128. 17. STINCHFIELD, F. E.; SINTON, W. A. - Criteria for spine fusion with the use of " $H$ " bone graft following disc removal. Arch. Surg., 65:542, 1952. 18. TUCKER, A. S. - Myelography of complete spinal obstruction. Am. J. Roentgenol., 76:248, 1956. 19. WILSON, J. N.; ILFELD, F. W. Manipulation of the herniated intervertebral disc. Am. J. Surg., 83:173, 1952.20. WOOLSEY, R. D.; TSANG, J. L. - A review of three hundred cases of protruded intervertebral discs treated surgically. J. Internat. Coll. Surgeons, 18:456, 1952.

Serviço de Neurologia da Escola Punlista de Medicina - Caixa Postal 5496 São Paulo, Brasil. 